\title{
Impact of restrictions due to COVID-19 on a quality-assured screening colonoscopy program
}

\section{다 $(9)$}

\author{
Authors \\ Anna Hinterberger ${ }^{1,2}$, Lena Jiricka ${ }^{1,2}$, Elisabeth A. Waldmann ${ }^{1,2}$, Daniela Penz ${ }^{1,2}$, Barbara Majcher ${ }^{1,2}$, Arno Asaturi $^{1,2}$, \\ Aleksandra Szymanska1,2, Lisa Rockenbauer ${ }^{1,2}$, Arnulf Ferlitsch², Michael H. Trauner ${ }^{1,2}$, Monika Ferlitsch ${ }^{1,2}$
}

Institutions

1 Division of Gastroenterology and Hepatology, Department of Internal Medicine III, Medical University of Vienna, Austria

2 Quality Assurance Working Group, Austrian Society for Gastroenterology and Hepatology, Austria

submitted 10.11.2020

accepted after revision 4.3.2021

\section{Bibliography}

Endosc Int Open 2021; 09: E1315-E1320

DOI 10.1055/a-1497-1123

ISSN 2364-3722

(C) 2021. The Author(s).

This is an open access article published by Thieme under the terms of the Creative Commons Attribution-NonDerivative-NonCommercial License, permitting copying and reproduction so long as the original work is given appropriate credit. Contents may not be used for commercial purposes, or adapted, remixed, transformed or built upon. (https://creativecommons.org/licenses/by-nc-nd/4.0/)

Georg Thieme Verlag KG, Rüdigerstraße 14,

70469 Stuttgart, Germany

Corresponding author

Univ.-Prof. Dr. Monika Ferlitsch, Div. of Gastroenterology and Hepatology, Dept. of Internal Medicine III, Medical University of Vienna, Waehringer Guertel 18-20, 7i, 1090 Vienna, Austria

Fax: $+43(1) 4040047350$

monika.ferlitsch@meduniwien.ac.at

\section{ABSTRACT}

Background and study aims On February 25, 2020, the first patient was diagnosed with COVID-19 in Austria. On March 16, 2020, the Austrian government imposed restrictions and subsequently the Austrian Medical Association recommended minimizing screening examinations in compliance with government restrictions. The aims of this study were to evaluate the impact of this recommendation on the number of colonoscopies performed weekly and detection of non-advanced adenomas, advanced adenomas (AA) and colorectal cancer (CRC) and to calculate how many undetected adenomas could have developed into CRC.

Methods We analyzed the number of colonoscopies and pathological findings within a quality assured national colorectal cancer screening program before the COVID-19 pandemic (March 1, 2019 to September 1, 2019, Period 1) and compared those rates to months during which access to colonoscopy was limited (March 1, 2020 and September 1, 2020, Period 2) with a Wilcoxon-rank-test and a chi-square test.

Results A total of 29,199 screening colonoscopies were performed during Period 1 and 24,010 during Period 2. The mean rate of colonoscopies per week during Period 1 was significantly higher than during Period 2 (808,35 [SD = $163,75]$ versus 594,50 [SD $=282,24], P=0.005)$. A total of 4,498 non-advanced adenomas were detected during Period 1 versus 3,562 during Period $2(P<0.001)$. In total 1,317 AAs and 140 CRCs were detected during Period 1 versus 919 AAs and 106 CRCs during Period 2. These rates did not differ significantly $(P=0.2$ and $P=0.9)$.

Conclusions During the COVID-19 crisis, the number of colonoscopies performed per week was significantly lower compared to the year before, but there was no difference in the detection of CRCS and AAs.

\section{Introduction}

In December 2019, a respiratory disease spread in Wuhan, China caused by the novel Coronavirus SARS-CoV-2. The disease, COVID-19, shows mild symptoms in most cases, but severe cases with fever, cough, breathing difficulties, and lethal out- comes can occur. A study from Wuhan showed that invasive medical ventilation was required in $2.3 \%$ of patients with SARS-CoV-2 and $1.4 \%$ died [1,2].

A few months later, the situation also became critical in Europe, with Italy the first country to be severely affected with approximately 30,000 deaths (as of March 7, 2020) [3]. It did not 
take long until the virus spread to Austria. In the federal state Tyrol, the first two patients were tested positive for SARS-CoV2 on February 25 [4]. Due to increasing numbers of SARS-CoV-2 infections, lockdown restrictions were implemented in Austria during week 12 (March 16, 2020). The first relaxation of the initial restrictions occurred during week 16. In March the World Health Organization declared the disease a pandemic. Therefore, politicians and medical facilities in Austria took measures to keep the infection rate as low as possible [5-8].

Particularly in endoscopic units, the risk of infection for patients and medical healthcare professionals is high due to the fact that the virus spreads via droplets and aerosol, which are easily released during upper gastrointestinal endoscopy. Also during colonoscopy, the risk of infection needs to be considered because of the presence of viral RNA in stool samples; even intact virus has been demonstrated in some studies. In a study of Wang et al., two of 20 patients who tested positive for SARS-CoV-2 had live virus in their stool samples [9]. In a study of Wölfel et al., the concentration of viral RNA in stool was nearly similar to the concentration in sputum, but no live SARS-CoV-2 virus was observed [10]. Therefore, a fecal-oral route of transmission has been discussed in several studies $[11,12]$.

On March 16, the government of Austria implied restrictions with lockdown of public life [13]. These restrictions also had a severe impact on medical facilities. Personal protective equipment (PPE) was required to protect professionals and patients, and direct patient contact was limited to urgent, vitally necessary examinations $[14,15]$.

Therefore, the Austrian medical association recommended minimizing screening colonoscopy $[16,17]$.

Screening colonoscopy, however, is the gold standard examination in prevention of colorectal cancer (CRC) [18]. During the procedure, precancerous lesions, which can be non-advanced, defined as lesions $<1 \mathrm{~cm}$ with no advanced characteristics or advanced adenomas (AA), defined as adenomas $\geq 1 \mathrm{~cm}$ with high-grade dysplasia, or tubulovillous or villous characteristics can be removed $[19,20]$.
The aim of this study was to analyze whether screening colonoscopy continued and how many non-advanced, advanced adenomas, and carcinomas were detected during the COVID19 crisis. Furthermore, the aim was to evaluate how many of the undetected adenomas could potentially have develop into CRC within 1 year.

\section{Methods}

\section{Data source}

This study was a retrospective cohort of patients who underwent screening colonoscopy. Data stem from the databases of the "Certificate of quality for screening colonoscopy," which is a quality assurance initiative in the framework of a national CRC screening program. It was funded in 2007 by the Austrian society of Gastroenterology and Hepatology, the Austrian Federation of the Statutory Insurance Institutions and the Austrian Cancer Aid. About $50 \%$ of all endoscopists, including internists, surgeons, and specialists in gastroenterology, who perform colonoscopies in hospitals or in private practices participate in the program.

Details of screening colonoscopies were submitted via an online form, which included morphology of the polyps, polypectomy technique, and histological details based on pathology reports. To verify the accuracy of data submission, annual spot checks were made.

\section{Study cohort}

For this study, the number of colonoscopies performed per week in 2019 and 2020 were compared, between March 1 and September 1 . In total, data from 184 endoscopic centers were included.

\section{Statistical analysis}

Counts of colonoscopies performed per week from March 1, 2019 to September 1, 2019 (Period 1) and from March 1, 2020 to September 1, 2020 (Period 2) are presented as total numbers and the percentage difference between Period 1 and 2 is dis-

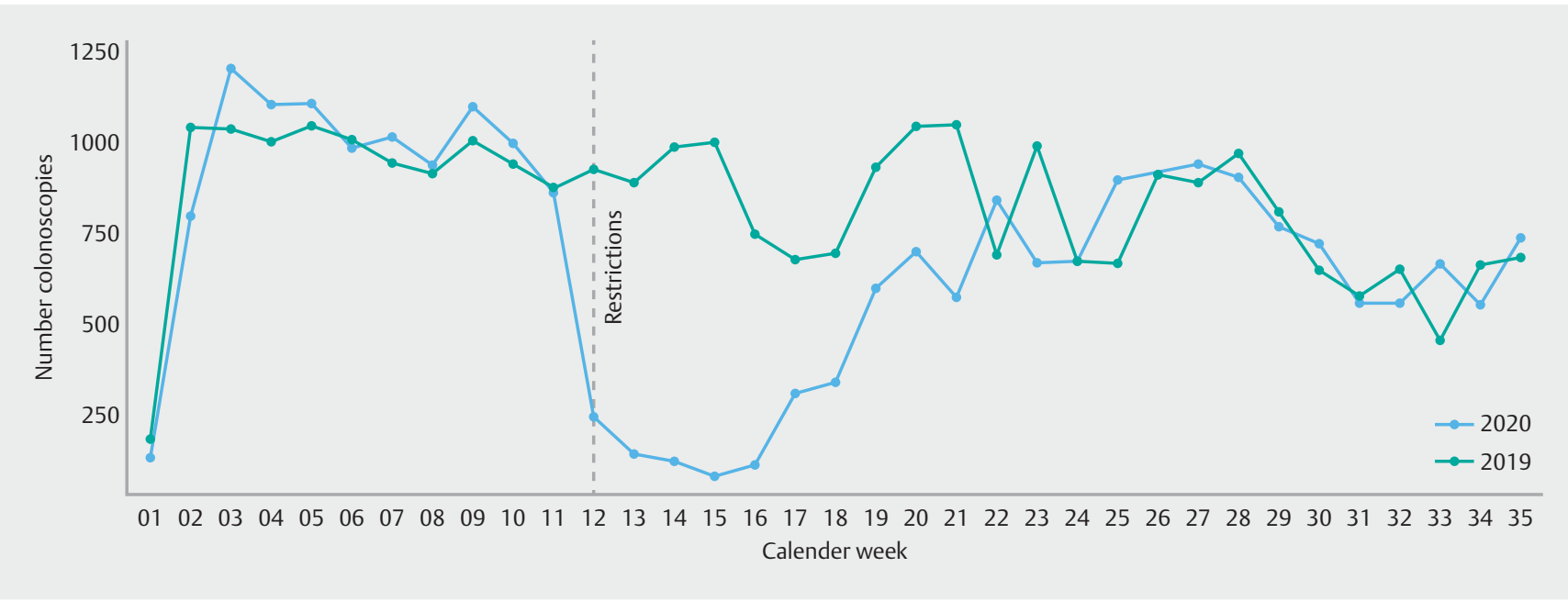

Fig. 1 Numbers of colonoscopies performed per week. 
played. To illustrate the course of colonoscopies performed weekly and pathological findings throughout the year, figures show total numbers from January 1, to September 1, in 2019 and 2020. Furthermore, colonoscopies per week are displayed as means for better comparability. The means were calculated by dividing counts of colonoscopies each week by all colonoscopies from March 1 until September 1 in 2019 and 2020. Those rates were compared with a Wilcoxon-rank-test. Pathological findings were analyzed by defining three types of diagnoses: non-advanced adenomas, advanced adenomas, and CRC. Advanced adenomas were defined as adenoma $\geq 10 \mathrm{~mm}$ or with high-grade dysplasia, or villous or tubulovillous histology and serrated lesions $\geq 10 \mathrm{~mm}$ or with dysplasia. Non-advanced adenomas were defined as adenomas without advanced characteristics. Total counts and non-advanced adenoma detection rate, advanced adenoma detection rate and colorectal cancer detection rate were compared between Periods 1 and 2 by using a chi-square test. Moreover, potential CRC cases were analyzed with a transmission rate of $4 \%$ for non-advanced adenomas developing into advanced adenomas and $3.1 \%$ of advanced adenomas developing into CRCs [21]. Statistical analyses were performed with $\mathrm{R}$.

\section{Results}

\section{Performed colonoscopies per week}

In total, 29,199 screening colonoscopies were performed during Period 1 versus 24,010 during Period 2. During week 11, 864 colonoscopies were performed, whereas in week 12 , the week during which the lockdown restrictions were implemented, the count was 244. During Period 1 the highest count $(n=$ 1.048) of colonoscopies performed weekly was in week 21 and the lowest was in week $33(n=453)$ whereas during Period 2, highest count was during week $10(n=996)$ and the lowest during week $15(n=80)$, with $92 \%$ fewer colonoscopies during Period 2 than during Period 1.

The mean rate of colonoscopies performed per week during Period 1 (2019) was 808.35 (SD = 163.75) versus 594.50 (SD = 282.24) during Period 2 (2020). A two-sample Wilcoxon-ranktest showed a significant difference between the means $(P=$ 0.005). As $>$ Fig. 1 shows, between weeks 11 and 12, the number of screening colonoscopies fell rapidly and slowly increased again until week 16 . Despite the increase during week 16 , the discrepancy between the numbers of screening examinations between 2019 and 2020 is high.

The number of colonoscopies performed per week during Periods 1 and 2 are shown as absolute counts in $>$ Fig. 1 and as percentage difference $>$ Table 1 .

\section{Pathological findings}

Total counts of pathological diagnoses during Period 1(2019) and Period 2 (2020) per week are shown in > Fig. 2.

In total, during Period 1, 4,498 non-advanced adenomas were detected, versus 3,562 during Period $2(P<0.001)$. As - Fig. 2 shows, the count of detected non-advanced adenomas fell beginning during week 12 and rose again during week 16 . Regarding advanced adenomas, counts did not fall as quickly
- Table 1 Colonoscopies performed per week.

\begin{tabular}{|c|c|c|c|}
\hline Week & 2019 & 2020 & Difference (\%) 2019 vs. 2020 \\
\hline 10.00 & 944.00 & 996.00 & -5.51 \\
\hline 11.00 & 870.00 & 864.00 & 0.69 \\
\hline 12.00 & 926.00 & 244.00 & 73.65 \\
\hline 13.00 & 888.00 & 145.00 & 83.67 \\
\hline 14.00 & 987.00 & 120.00 & 87.84 \\
\hline 15.00 & 1000.00 & 80.00 & 92.00 \\
\hline 16.00 & 746.00 & 114.00 & 84.72 \\
\hline 17.00 & 676.00 & 309.00 & 54.29 \\
\hline 18.00 & 697.00 & 338.00 & 51.51 \\
\hline 19.00 & 934.00 & 593.00 & 36.51 \\
\hline 20.00 & 1044.00 & 700.00 & 32.95 \\
\hline 21.00 & 1048.00 & 576.00 & 45.04 \\
\hline 22.00 & 685.00 & 843.00 & -23.07 \\
\hline 23.00 & 986.00 & 665.00 & 32.56 \\
\hline 24.00 & 675.00 & 673.00 & 0.30 \\
\hline 25.00 & 668.00 & 896.00 & -34.13 \\
\hline 26.00 & 907.00 & 912.00 & -0.55 \\
\hline 27.00 & 886.00 & 939.00 & -5.98 \\
\hline 28.00 & 973.00 & 901.00 & 7.40 \\
\hline 29.00 & 808.00 & 769.00 & 4.83 \\
\hline 30.00 & 650.00 & 719.00 & -10.62 \\
\hline 31.00 & 572.00 & 554.00 & 3.15 \\
\hline 32.00 & 650.00 & 553.00 & 14.92 \\
\hline 33.00 & 453.00 & 665.00 & -46.80 \\
\hline 34.00 & 660.00 & 553.00 & 16.21 \\
\hline 35.00 & 684.00 & 736.00 & -7.60 \\
\hline
\end{tabular}

as for non-advanced adenomas, but still, during Period 1 a total of 1,317 advanced adenomas were detected versus 919 during Period $2(P=0.2)$. During Period 1, 140 CRCs were detected versus 106 during Period $2(P=0.9)$. Details of pathological findings are shown in $>$ Table 2 and $>$ Fig. 2 . In Period 1 the rate of detection of non-advanced adenomas was $21 \%$ versus $23 \%$ during Period 2. Accordingly, the advanced adenoma detection rate was $6.3 \%$ during Period 1 versus $5.9 \%$ during Period 2. The rate of detection of CRCs was $0.7 \%$ during both periods.

Considering Period 1, $4 \%$ of 4.498 non-advanced adenomas $(n=179)$ could have developed into advanced adenomas and $3.1 \%$ of advanced adenomas could have developed into CRC within 1 year. Therefore, five CRC cases were prevented by resecting non-advanced adenomas during Period 1. During Period 2, 3,562 non-advanced adenomas correspond to four CRC cases prevented. Because $3.1 \%$ of 1,317 advanced adenomas 


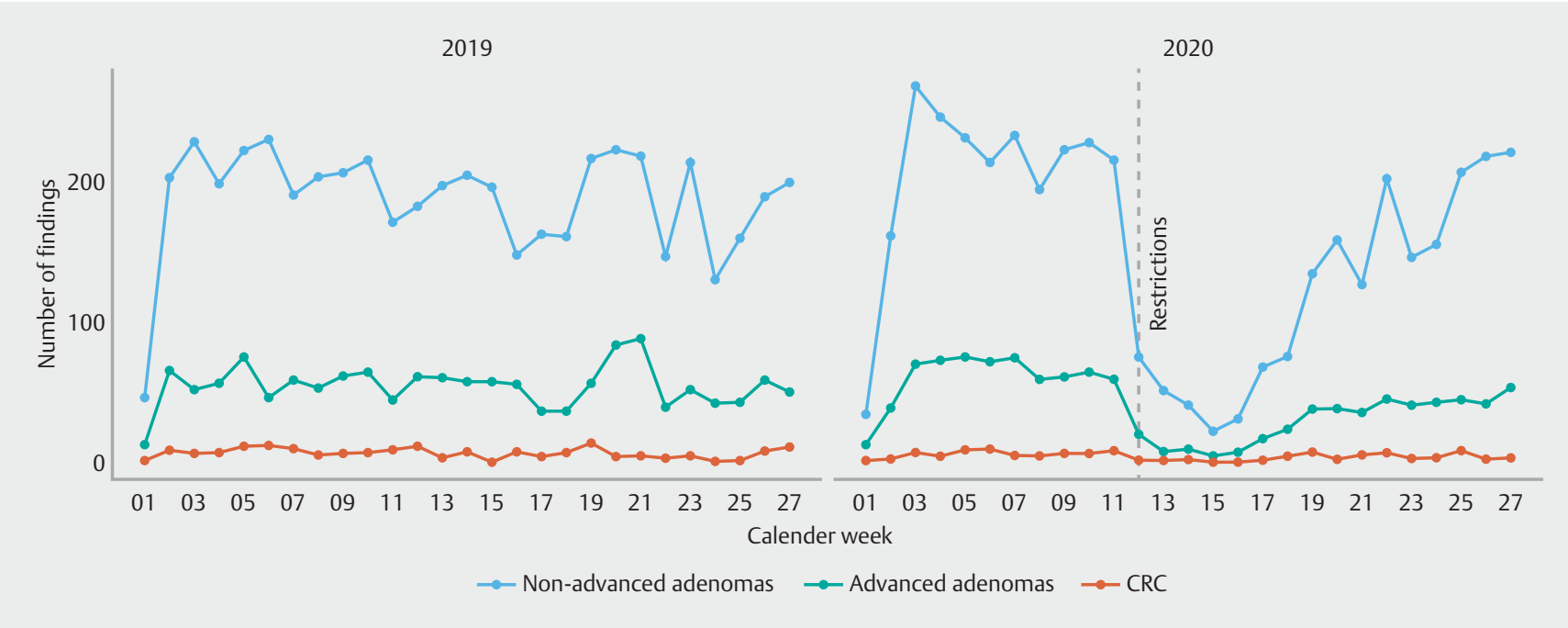

Fig. 2 Number of pathological findings per week.

- Table 2 Pathological findings during Periods 1 and 2.

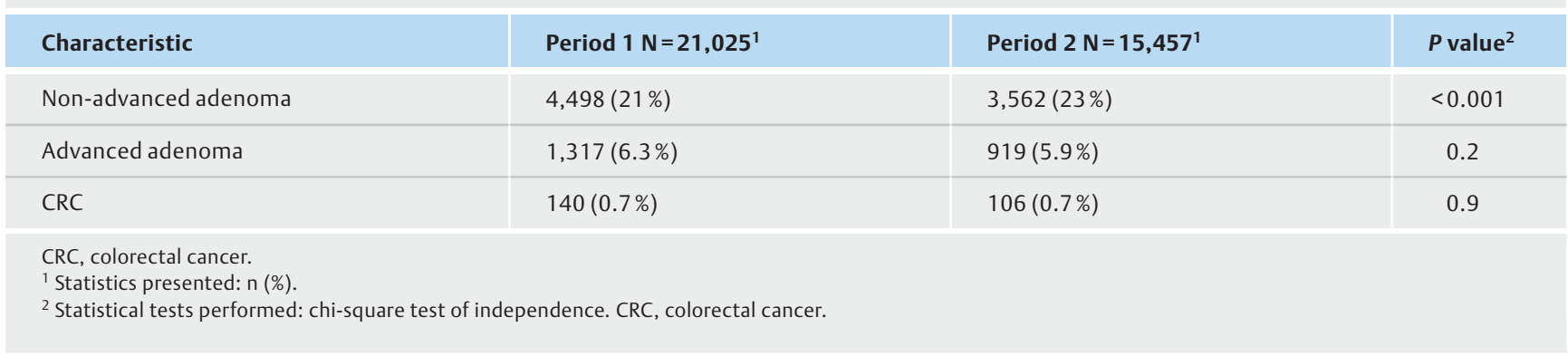

develop into CRC within 1 year, 41 CRC cases were prevented during Period 1 by resecting advanced adenomas versus 29 during Period 2. In total, during Period 1, 46 CRC cases were prevented, whereas during Period 2, 32 cases of CRC were prevented. The estimated number of CRC cases is shown in > Fig. 3.

\section{Discussion}

Screening colonoscopy is the gold standard examination for reducing colorectal cancer; however, preventive examinations were largely discontinued during the corona pandemic in Austria. The effect of such restrictions on screening examinations has so far remained unknown $[17,22]$.

With a total of 53,209 screening examinations performed by 184 endoscopists, this study provides data on how many precancerous lesions and CRCs were detected during the COVID19 pandemic compared to the year before and how many lesions were potentially missed due to restrictions. Following the restrictions during week 12 , the number of screening colonoscopies decreased by more than $90 \%$. From March 1 to September 1, 2019 the mean number of colonoscopies performed per week was 808 (SD=163.75), and therefore, significantly higher than in the same period of time in 2020 during which the mean was $594(S D=282.24)(P=0.005)$. These results show that in

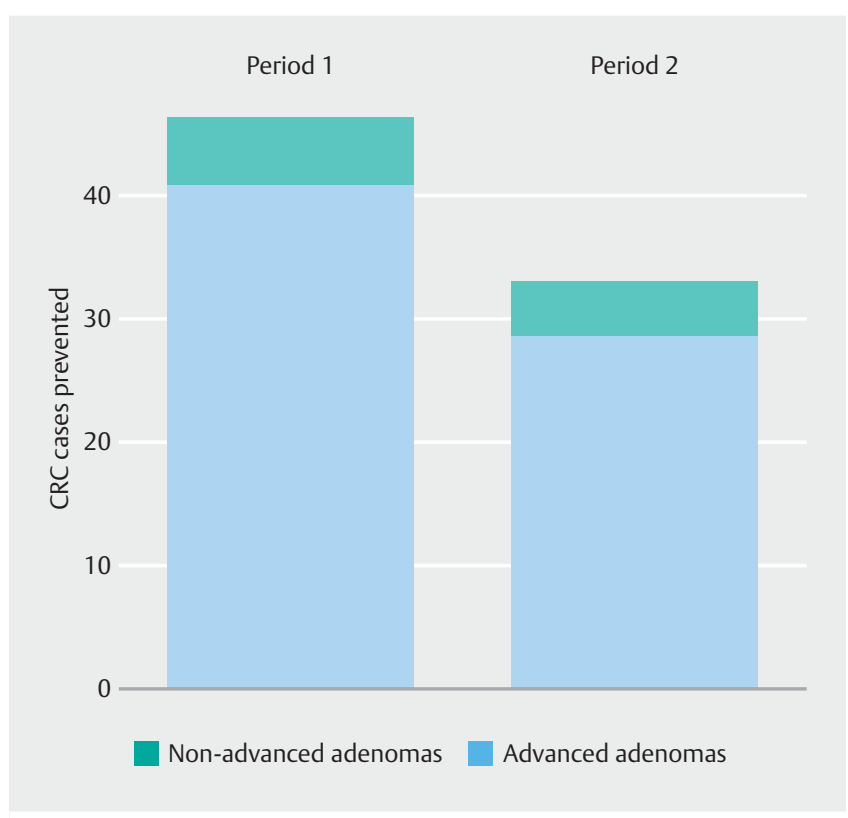

Fig. 3 Estimated number of CRC cases prevented.

2020, there were actually fewer colonoscopies performed than in 2019. 
The number of detected non-advanced adenomas was much lower in 2020 than in $2019(P<0.001)$. Interestingly, the number of advanced adenomas and CRCs detected in 2020 was not significantly lower than in 2019. The rate of CRC cases might not be lower because patients had symptoms and still underwent colonoscopy despite restrictions. Also, advanced adenomas may cause symptoms if they are large, and therefore, cause pain or blood in the stool. However, as a study of Brenner et al. shows, $4 \%$ of all non-advanced adenomas develop into advanced adenomas and $3.1 \%$ of advanced adenomas develop into CRC [21]. Therefore, in our study, a total of 46 CRCs were prevented during Period 1 versus 33 during Period 2. This is a gap of 13 prevented CRCs when comparing Period 1 and Period 2. These numbers need to be monitored further to make a statement about potentially increasing colorectal cancer incidence and CRC-related mortality. After week 17, the number of screening colonoscopies began to increase as the government eased the initial restrictions during week 16 . To ensure the protection of patients and examiners, the Austrian Association of Medicine started a campaign in the beginning of April in which doctors received CPA/FFP2 masks, classic masks, disinfectant, gloves, and protective glasses for free once a week. Despite the resumption of screening examinations, there is a gap of 5,189 screening colonoscopies when comparing 2019 and 2020 and it is uncertain how, if, and when all the postponed colonoscopies can be performed. In a paper published in May 2020 , it was noted that it is unclear whether there would be any consequences if colonoscopies were temporarily cancelled and if the incidence of CRC might rise. There were mainly concerns about patients with positive fecal occult blood tests (FOBTs) or fecal immunochemistry tests (FITs) requiring screening colonoscopy, because there could be a backlog due to the large number of endoscopies that need to be performed. Another reason for a potential increase in CRC rates, mentioned in this paper, is that patients do not keep their surveillance appointments because they are afraid of acquiring COVID-19 [23]. A study done in the United States in 2018 showed that discontinuing elective endoscopies would increase the mortality rate by $6.5 \%$ over 6 months [24]. As of September 2020, screening colonoscopies had been paused for 3 to 4 months in Austria and most medical facilities had resumed elective examinations. It is difficult to say whether this pause will have an impact, especially because during the crisis, CRCs and advanced adenomas were nevertheless removed and cancer prevention did not stagnate completely.

In a recent statement by the European Society of Gastrointestinal Endoscopy (ESGE), it was pointed out that the capacity for endoscopic procedures should be increased as much as possible and, at the same time, the risk of infection for patients and doctors/medical staff must be kept as low as possible to resume the "normal" clinical everyday life or to process the postponed examinations. ESGE also recommends that patients with a positive FIT or FOBT within an organized screening program be given high priority and undergo screening colonoscopy (as of July 2020) during the so called "post-lockdown-phase" [25]. In Austria, there is no nationwide organized screening program for colorectal cancer, so it is not clear how to proceed.

\section{Conclusions}

In conclusion, during the COVID-19 pandemic in 2020, significantly fewer screening colonoscopies were performed as compared to 2019, which should be compensated for by increasing endoscopy capacity in the future. Nevertheless, our study showed that restrictions so farhave had no significant impact on the rates of detection of advanced adenomas and CRCs. Accordingly, no increased incidence or mortality rates from CRC are expected, if the postponed colonoscopies are now performed.

\section{Competing interests}

The authors declare that they have no conflict of interest.

\section{References}

[1] Huang C, Wang Y, Li X et al. Clinical features of patients infected with 2019 novel coronavirus in Wuhan, China. Lancet 2020; 395: 497-506

[2] Guan W, Ni Z, Hu Y et al. Clinical characteristics of Coronavirus Disease 2019 in China. NEJM 2020; 382: 1708-1720

[3] Gralnek I, Hassan C, Beilenhoff U et al. ESGE and ESGENA Position Statement on gastrointestinal endoscopy and the COVID-19 pandemic. Endoscopy 2020; 52: 483-490

[4] https://orf.at/stories/3155602/ [25.02.2020]

[5] https://orf.at/corona/stories/3157533/ [20.07.2020]

[6] https://www.tagesschau.de/ausland/oesterreich-lockerungen-101. html [14.04.2020]

[7] https://oesterreich.orf.at/stories/3049163/ [17.05.2020]

[8] https://www.euro.who.int/en/health-topics/health-emergencies/ coronavirus-covid-19/news/news/2020/3/who-announces-covid-19outbreak-a-pandemic [12.03.2020]

[9] Wang W, Xu Y, Gao R et al. Detection of SARS-CoV-2 in different types of clinical specimens. JAMA 2020; 323: 1843-1844

[10] Wolfel R, Corman VM, Guggemos W et al. Virological assessment of hospitalized patients with COVID-2019. Nature 2020; 581: 465-469

[11] Perisetti A, Gajendran M, Boregowda U et al. COVID-19 and gastrointestinal endoscopies: Current insights and emergent strategies. Digest Endosc 2020; 32: 715-722

[12] Gu J, Han B, Wang J et al. COVID-19: Gastrointestinal manifestations and potential fecal-oral transmission. Gastroenterol 2020; 158: 1518-1519

[13] Im Internet (aufgerufen am 04.04.2020): https://www.servustv.com/ ausgangsbeschraenkungen-in-oesterreich-uebersicht/

[14] http://www.salute.gov.it/imgs/C_17_notizie_4702_0_file.pdf [07.05.2020]

[15] Joint Advisory Group et al. Advice for Endoscopy Teams during COVID-19. British Society of Gastroenterology; 2020

[16] https://www.sozialministerium.at/Informationen-zum-Coronavirus/ Coronavirus-Rechtliches.html [11.08.2020]

[17] https://www.aerztekammer.at/documents/261766/548282/202004-15+Empfehlungen+Ordinationen+-+Anlage_Empfehlung+für+Ordinationen+in+der+Pandemie.pdf/9c041aed-4fd9-985c-154d9c1d9cd39c16? $t=1587036607429$ [16.04.2020]

[18] Zauber AG, Winawer SJ, O’Brien MJ et al. Colonoscopic polypectomy and long-term prevention of colorectal-cancer deaths. N Engl J Med 2012; 366: 687-696 
[19] Dekker E, Rex D et al. Advances in CRC prevention: screening and surveillance. Gastroenterol 2018; 154: 1970-1984

[20] Click P, Pinsky P, Hickey T et al. Association of colonoscopy adenoma findings with long-term colorectal cancer incidences. JAMA 2018; 319: 2021-2031

[21] Brenner $\mathrm{H}$, Altenhofen L, Stock C et al. Natural history of colorectal adenomas: birth cohort analysis among 3.6 million participants of screening colonoscopy. Cancer Epidemiol Biomarkers Prev 2013; 22: 1043-1051

[22] Schreuders E, Ruco A, Rabeneck L et al. Colorectal cancer screening: a global overview of existing programmes. Gut 2015; 64: 1637-1649
[23] Gralnek I, Hassan C, Dinis-Ribeiro M et al. COVID-19 and endoscopy: implications for healthcare and digestive cancer screening. Nat Rev Gastroenterol Hepatol 2020; 13: 1-3

[24] Peery A, Crockett S, Murphy C et al. Burden and cost of gastrointestinal, liver, and pancreatic disease in the United States: update 2018. Gastroenterol 2019; 156: 254-272

[25] Gralnek I, Hassan C, Beilenhoff U et al. ESGE and ESGENA Position Statement on gastrointestinal endoscopy and COVID-19: An update on guidance during the post-lockdown phase and selected results from a membership survey. Endoscopy 2020; 52: 891-898 